\title{
TUBERCULOSE ILEOCECAL ISOLADA SIMULANDO NEOPLASIA MALIGNA E DOENÇA DE CROHN
}

S.H. Bromberg, S. Faroud*, F.F. de Castro, N. Morrone, A.C. de Godoy, l.C.M. França

TrabalhorealizadonoSenviçoenaPós-Graduaçãoem GastroenterologiaCirúrgicadoHospital doSenvidorPúblicoEstadual-FranciscoMoratodeOliveira,SãoPaulo,SP.

RESUMO - OBjetivo. No Brasil, a tuberculose ileocecal isolada, na ausência de tuberculose pulmonar ativa ou cicatrizada, é rara, sendo freqüentemente confundida com neoplasia e a doença de Crohn.

Métodos. Os autores apresentam oito doentes com essas características, tratados por cirurgia, em período de 20 anos. A dor abdominal na fossa ilíaca direita foi o principal sintoma (100\%), seguido da presença de perda de peso $(62,5 \%)$, febre, náuseas e vômitos e fadiga em $50 \%$ dos doentes. Sete enfermos (87,5\%) apresentaram massa palpável na fossa ilíaca direita. A duração média dos sintomas foi de 14,7 meses, variando de 5 a 36 meses. Todas as lesóes foram detectadas pelo enema opaco e mimetizavam aspecto neoplásico. A colonoscopia foi realizada em três doentes, evidenciando lesões sub-oclusivas da região íleocecal em dois; as biópsias foram sugestivas de tuberculose, não sendo possível diferenciá-las da doença de Crohn.

Resultados. Seis enfermos com diagnóstico presuntivo de neoplasia foram submetidos a cirurgia eletiva, realizando-se neles a colectomia direita clássica. Os outros dois, com suspeita de tuberculose, foram operados na vigência de obstrução intestinal, sendo submetidos a ressecção limitada (ileocecectomia). 0 estudo anatomopatológico estabeleceu o diagnóstico final pelo encontro de necrose caseosa e de bacilos álcool ácido-resistentes no intestino ou nos linfonodos. A evolução pós-operatória foi satisfatória, com pequena morbidade resolvida clinicamente. Posteriormente, todos os doentes receberam o esquema tríplice por 12 meses, variando a droga de acordo com a época do tratamento.

Conclusão. Apesar de rara, a tuberculose hipertrófica ileocecal isolada freqüentemente é confundida com neoplasias e com a doença de Crohn, devendo ser lembrada no diagnóstico diferencial das lesões localizadas no quadrante inferior direito do abdome.

UniteRMOS: Tuberculose abdominal. Tuberculose intestinal. Tuberculose ileocecal. Tuberculose extra-pulmonar.

\section{INTRODUÇÃO}

Nos últimos anos, a tuberculose intestinal voltou a preocupar seus estudiosos por apresentar expressiva recrudescência. Países desenvolvidos constatam-na em número expressivo de imigrantes oriundos de nações, onde a tuberculose é prevalente, mas vêem também seus doentes imunodeprimidos assolados pela doença. No Brasil, a tuberculose intestinal está, geralmente, associada à presença de lesões pulmonares, ativas ou cicatrizadas, ao passo que em áreas não prevalentes, o número de lesões intestinais

\footnotetext{
${ }^{*}$ Correspondência

Rua ConselheiroBrotero, 1505-Coni. 51 Cep:01232-010-SãoPaulo-SP Fone: $3826-2600$
}

não acompanhadas das pulmonares é maior'. Nesta eventualidade, a tuberculose intestinal freqüentemente é confundida com outras afecções, como as neoplasias e a doença de Crohn, o que dificulta, em muito, a terapêutica a ser instituída ${ }^{2-4}$. Em período de vinte anos, tivemos a oportunidade de conduzir o tratamento cirúrgico de oito doentes com tuberculose isolada da região ileocecal, sem evidências de tuberculose extra-intestinal, vivenciando dificuldades diagnósticas e terapêuticas, razões do presente estudo.

\section{Métodos}

Reunimos oito portadores de tuberculose intestinal isolada, tratados no Serviço de Gastroenterologia Cirúrgica do HSPE-
FMO, no período de 1969 a 1989. Os dados deste estudo foram colhidos por entrevistas com os doentes, por telefone ou nos ambulatórios, e por consultas aos respectivos prontuários. Seis pacientes eram do sexo masculino, cinco eram brancos e três negros; a média de idade foi de 53,1 anos (38 a 66 anos). Todos os doentes negavam doença tuberculosa anterior e apresentavam radiografias normais dos campos pleuro-pulmonares.

A duração média dos sintomas foi de 14,7 meses, variando de 5 a 36 meses. Todos apresentaram dor abdominal e somente um deles não tinha massa abdominal palpável no quadrante inferior direito. 0 quadro clínico completo apresentado pelos doentes pode ser observado na Tabela I. 
BROMBERG SH ET AL.

Todos tinham hemossedimentação elevada (acima de $30 \mathrm{~mm} / \mathrm{h}$ ) e anemia, com hemoglobina média de 9, I g/ (7,2a I I,3 g/ I). O PPD, realizado em seis enfermos, foi positivo em três deles.

As lesões foram evidenciadas pelo enema opaco em todos os doentes, predominando imagens de efeito de massa intraluminal e deformidade ileocecal, sugestivos de neoplasias. (Figura I).

A colonoscopia, de introdução mais recente, foi realizada somente em três deles (37,5\%). Dois desses doentes mostravam no ceco vegetações lobuladas revestidas por mucosa aparentemente íntegra, porém edemaciada, acompanhadas por deformidades da base cecal causadas por compressões extrínsecas; o ileo terminal mostrava-se com paredes espessadas e luz diminuída. O terceiro exibia lesão ulcerada e vegetante no nível da válvula ileocecal, com mucosa adjacente de aspecto finamente granular, sugerindo 0 diagnóstico de neoplasia. Nos dois primeiros doentes, as biópsias colhidas evidenciaram a presença de reação inflamatória crônica granulomatosa; no terceiro, notou-se apenas focos superficiais de ulceração mucosa, revestida por fibrina e neutrófilos, com proliferação fibroblástica e capilar. Todos os enfermos tiveram seus diagnósticos de certeza somente após estudo anatomopatológico das peças ressecadas, que mostraram granulomas com focos de necrose de caseificação no intestino em quatro e a mesma lesão nos linfonodos dos demais doentes. A presença de bacilos álcool-ácido-resistentes foi positiva em quatro dos espécimes estudados.

\section{Resultados}

Em seis doentes (75\%) a cirurgia foi indicada na presunção de doença maligna, sendo submetidos à colectomia direita oncológica clássica. Os dois restantes, com processos sugestivos de Crohn ou tuberculose nas biópsias a que foram submetidos desenvolveram quadros oclusivos, sendo por isso submetidos a tratamento cirúrgico com o diagnóstico de doença granulomatosa oclusiva. Nesses dois enfermos (25\%) praticou-se a ileocecectomia (exerese do ceco e de pequeno segmento do ileo terminal), sendo o trânsito intestinal reconstituído por anastomose do ileo com o colo ascendente. As lesões eram vegetantes em cinco e úlcero-vegetantes em três doentes. Nos primeiros, a maior das lesões media $10 \times 4 \mathrm{~cm} \mathrm{e} \mathrm{a}$ menor $6 \times 3 \mathrm{~cm}$.

Não houve óbitos; três pacientes apresentaram infecção da parede abdominal no nível da cicatriz operatória e em um deles foi constatada pequena fístula estercorácea, que cicatrizou espontaneamente em quinze dias. Todos receberam tratamento complementar com esquema tríplice, que variou com a época. Os mais antigos foram tratados com etambutol, ácido aminosalićlico, estreptomicina ou rifampicina (RMP), sempre associados à isoniazida $(\mathrm{INH})$ e, nos mais recentes, foi incorporado o emprego da pirazinamida (PZA).

\section{Discussão}

A presença de tuberculose intestinal na ausência de comprometimento extra-digestivo não é sinônimo de tuberculose pri- mária. Em estudo realizado em 23 pacientes com lesões digestivas, encontramos 13\% deles com lesões isoladas, o que não nos autorizou a reconhecê-las como primárias 5 . Somente após encontrar o complexo primário abdominal, Sodré et al. classificaram como portadores de doença primária digestiva quatro de 2.408 tuberculosos necropsiados 6 . Assim, a constatação de tuberculose intestinal na ausência de comprometimento pulmonar radiológico deve ser interpretada como tuberculose isolada. A real explicação de sua origem é difícil, sendo plausível supor a patogenia secundária, justificada pelo inadequado reconhecimento do foco primário pulmonar. Alguns estudio- 
sos chamam atenção para a possibilidade da difusão linfática retrógrada, oriunda de linfonodos acometidos por ocasião da instalação da doença primária, quer abdominal quer torácica ${ }^{3}$.

$\mathrm{Na}$ quase totalidade dos casos a infecção é devida ao Mycobacterium tuberculosis, mesmo em países onde a tuberculose bovina é prevalente ${ }^{2,4}$. No Brasil, é excepcional a constatação da variedade bovina como agente etiológico de lesões intestinais.

O diagnóstico de tuberculose está condicionado à presença de uma das condições: cultura e inoculação positivas, demonstração histológica de bacilos álcoolácido resistentes na lesão, presença de necrose caseosa e evidências histológicas específicas em linfonodos regionais ${ }^{7}$.

A dificuldade do reconhecimento destas lesões em material de biópsia endoscópica é habitual, sendo mais uma vez confirmada nos doentes deste estudo. Mesmo em peças cirúrgicas, o encontro de bacilos e de necrose caseosa não é freqüente, embora sejam encontrados comumente nos linfonodos', como pudemos constatar. Quando ausentes, o diagnóstico diferencial com a moléstia de Crohn é extremamente difícil.

No entanto, a presença de metaplasia pilórica e de granulomas confluentes e grandes, com fibrose circunjascente, bem como sua presença nos linfonodos quando ausentes no intestino, são mais encontrados na tuberculose 7 . Por outro lado, nota-se na doença de Crohn, hiperplasia folicular transmural, granulomas pequenos e discretos, maior número de fissuras e de fístulas internas, bem como segmentos estenosantes mais longos ${ }^{7}$. Seguramente, todos esses parâmetros não serão encontrados nas biópsias endoscópicas.

O quadro clínico é inespecífico, sendo os sintomas compatíveis com múltiplas doenças intestinais. A dor abdominal, a presença de tumor palpável e o emagrecimen- to, principais sintomas dos enfermos deste estudo, são relatados freqüentemente ${ }^{1,4,8}$. Quando presente a diarréia, dificuldades maiores são encontradas no diagnóstico diferencial com a doença de Crohn.

Dois deles apresentaram sintomatologia crônica incaracterística - 29 e 36 meses, respectivamente. Tal fato também é relatado pelos estudiosos, que chamam a atenção para quadros clínicos de longa duração, com menor e inconstante sintomatologia dolorosa,não acompanhada de sinais físicos ${ }^{9}$. A presença de massa abdominal palpável, presente em $50 \%$ a $80 \%$ dos doentes, é comum e sugere processo neoplásico,8. Tal fato, presente em sete $(87,5 \%)$ dos doentes deste estudo, contribuiu para que seis deles fossem operados com suspeita de neoplasia maligna.

Alguns sinais radiológicos como o encurtamento do colo ascendente, a perda do ângulo normal entre o ileo e o ceco e alterações inflamatórias localizadas do colo direito são mais sugestivas de tuberculose ${ }^{10,11}$. Na moléstia de Crohn é característica a ocorrência simultânea, no mesmo segmento intestinal, de dois ou três tipos de alterações, que correspondem a diferentes estádios da doença na alça intestinal, bem como a presença de trajetos fistulosos" ${ }^{\prime}$. Segmentos estenosados de extensão maior que $3 \mathrm{~cm}$ são mais compatíveis com doença de Crohn. O diagnóstico diferencial com as neoplasias é difícil, como pudemos observar neste estudo, não se podendo esquecer de relatos que assinalam a presença de ambas moléstias ${ }^{\prime 2}$. Mais recentemente foram incorporados ao armamentário diagnóstico a ecografia e a tomografia computadorizada, não utilizados neste estudo. A ultra-sonografia detecta com facilidade espessamentos parietais e a linfadenite mesentérica; o espessamento é mais expressivo na moléstia de Crohn, por sua característica inflamação trans-mural ${ }^{10}$. A tomografia computadorizada é útil na identificação de espessamentos parietais do intestino e identifica facilmente a sempre presente distorção da válvula ileocecal e a adenopatia mesentérica" .

As lesões endoscópicas são representadas por segmentos estenosantes curtos, anulares, de consistência firme, como observados no íleo terminal de um dos nossos doentes. A válvula ileocecal mostra-se constantemente edemaciada e deformada. Os doentes estudados apresentaram predominantemente lesões de aspecto vegetante, capazes de mimetizar doença neoplásica, fato comumente observado ${ }^{4,8}$. Pode-se também observar ulcerações profundas, de 0, I a 2,0 cm, transversais. As úlceras observadas na doença de Crohn aparecem em mucosa aparentemente normal, diferindo da tuberculosa, onde a mucosa circunjacente mostra-se edemaciada, eritematosa e nodular ${ }^{13}$. Os granulomas e os bacilos tuberculosos localizam-se além da mucosa, ocupando camadas mais profundas da parede intestinal; múltiplas e repetidas biópsias endoscópicas no mesmo local, conseguem maior número de amostras teciduais contendo anormalidades sugestivas de tuberculose ${ }^{2,4,13}$. Nas peças cirúrgicas de lesões hipertróficas, como pudemos comprovar, o reconhecimento de bacilos e granulomas caseosos é mais freqüente.

Em princípio, tão logo diagnosticadas, as lesões intestinais devem ser tratadas clinicamente, consistindo a terapêutica no esquema oral composto pela INH $400 \mathrm{mg} / \mathrm{dia}$, RMP 600 mg/dia e PZA 2,0 g/dia, os dois primeiros mantidos no mínimo por seis meses e o último, somente nos dois primeiros meses de tratamento. Na maioria dos doentes deste trabalho, a terapêutica específica foi mantida por doze meses, como indicado na época. Mesmo pacientes com sub-estenoses apresentam resposta favorável ao tratamento clínico ${ }^{14}$.

Nas formas intestinais isoladas obstrutivas ou quando da impossibilidade diag- 
nóstica, está indicada a cirurgia, como realizado nos doentes deste estudo. A diferenciação entre tuberculose e doença de Crohn, mesmo com o abdome aberto, nem sempre é fácil. $\mathrm{O}$ encontro de pequenos nódulos serosos é observado na tuberculose e nunca estão presentes no Crohn. Quando eletiva, a conduta ideal é a ressecção, seguida de anastomose primária. Dispondo-se no pré-operatório do diagnóstico presuntivo de benignidade ou de tuberculose, e indicada a cirurgia por complicações da moléstia, as ressecções devem ser limitadas, realizando-se sempre que possível, a exerese ileocecal ao invés de colectomia direita, como realizado em dois dos doentes estudados. Derivações intestinais devem ser evitadas por favorecerem a instalação de alças cegas, com seu cortejo de proliferação da flora anormal e má-absorção? Evidências de processo tuberculoso nas margens da ressecção não favorecem a formação de fístulas pós-operatórias ${ }^{15}$.

O prognóstico dos portadores de lesões hipertróficas é extremamente favorável. Todos os doentes deste estudo foram acompanhados por, no mínimo, três anos, sendo considerados curados e tendo retornado às suas atividades habituais.

A tuberculose ileocecal isolada mimetiza freqüentemente o quadro das neoplasias malignas da região. Os achados radiológicos são inespecíficos e o diagnóstico préoperatório, baseado nas biópsias obtidas por colonoscopia, só é alcançado quando elas forem numerosas e colhidas repetidas vezes no mesmo local, fornecendo assim ao patologista melhores espécimes, capazes de apresentar dados patognomônicos da doença. Tão logo efetuado o diagnóstico a medicação específica deve ser prontamente instituída. $\mathrm{Na}$ impossibilidade diagnóstica nas formas obstrutivas agudas e nas sem resposta ao tratamento clínico, indicase cirurgia, recomendando-se sempre a exerese da lesão.

\section{SUMMARY}

\section{ISOLATED ILEOCECAL TUBERCULOSIS SI- MULATING MALIGNANT NEOPLASIA AND CROHN'S DISEASE}

BACKGROUND. Isolated ileo cecal involvement by tuberculosis in the absence of pulmonar disease is rare in Brasil, therefore causes a diagnostic dilemma as it mimics colonic malignancy and Crohn's disease.

Methods. Between 1969 and 1989, eight patients with isolated hypertrophic ileocecal tuberculosis were treated by surgery in the Gastroenterology Surgery Department of the HSPE-FMO. The most common complaint among them was abdo minal pain (100\%) with associated symptoms of weight loss (62.5\%); nausea, vomiting, fever and general weekness appeared in half of the patients. A right iliac fossa mass was present in seven (87.5\%) of them. The mean duration of symptoms was 14.7 month (range 5-36 months). In all eight patients chest $x$-rays were negative for tuberculosis. Barium contrast studies showed abnormalities in all cases, but these could not be distinguished from carcinoma. Colonoscopy was helpful in establishing the diagnosis of suboclusive lesions of the ileocecal regions in three patients. Tuberculosis diagnosis was suspected in two of them because ofr the presence of granulomas in colonic biopsy material .

Results. Six patients were submitted to elective right hemicolectomy. The two remaining with suspect of tuberculosis were operated with signals of intestinal occlusion, and underwent a limited ileocaecal resection. The positive diagnosis of intestinal tuberculosis was made in all the patients by identification of acid-fast bacilli and by the presence of caseating granulomas in intestinal or lymph node tissue on histological examination. The outcome in all of them was favo rable. They received treatment with three antituberculosis drugs over a twelve month period.

Conclusion. Hypertrophic ileocecal tuberculosis must still be considered in the differential diagnosis of abdominal pathology localized in the right lower quadrant. [Rev Ass Med Brasil 200।; 47(2): 125-8]

KEYwORDS: lleocecal tuberculosis. Abdominal tuberculosis. Intestinal tuberculosis. Extrapulmonar tuberculosis.

\section{REFERÊNCIAS}

I. Palmer KR, Path DH, Basran GS, Riordan JF, SilkDBA-Abdominal tuberculosis in urban Britain - a common disease. Gut 1985;26: 1296-1305.

2. Bhargava DK, Tandon HD, Chawla TC, Tandon BN, Kapur BML - Diagnosis of ileocecal and colonic tuberculosis by colonoscopy. Gastrointest. Endosc. 1985; 31:68-70.

3. Chen W, Leu S, Hsu H, Lin J, Lin T. Trend of large bowel tuberculosis and the relation with pulmonary tuberculosis. Dis Colon Rectum 1992; 35:189-92

4. Ferentzi CV, Sleck JO, Ali MA - Colonoscopic diagnosis and medical treatment of ten patients with colonic tuberculosis. Endoscopy 1988; 20:62-5.

5. Bromberg SH, Habr-Gama A, Lima MT et al. Tuberculose intestinal. Rev Paul Med 1974; 84:|43-5|

6. Sodré HA, Croce J, Faria CV. Tuberculose intestinal. Rev Hosp Clin Fac Med Univ S Paulo 1946; 1: 317-48.

7. Tandon HD, Prakash A - Pathology of intestinal tuberculosis and its distinction from Crohn's disease. Gut 1972; 13:260-9.

8. Kasulke RJ, Anderson WJ, Gupta SK, Gliedman ML - Primary tuberculous enterocolitis. Report of three cases and review of the literature. Arch Surg 1981; 116:110-13.

9. Kapoor VK, Sharma LK - Abdominal tuberculosis. Br J Surg 1988; 75:2-3.

10. Leder RA, Low VHS - Tuberculosis of the abdomen. Radiol. Clin North Am 1995;33: 69|-705

I I. Balthazar EJ, Gordon R, Hulnick D - Ileocecal tuberculosis: CT and radiologic evaluation. Am | Roentgenol. 1990; 1 54:499-503.

12. Tandon HD, Kapoor BML - Carcinoma of the colon associated with tuberculosis. Report of a case. Dis Colon Rectum 1974; 17:777-81.

13. Shah S, Thomas V, Mathan Metal. Colonoscopy study of 50 patients with colonic tuberculosis. Gut | 992;33:347-5 |.

14. Anand BS, Nanda R, Sachdev GK - Response of tuberculous stricture to antituberculous treatment. Gut 1988; 29:62-9.

15. Veeragandham RS, Lynch FP, Canty TG, Collins DL, Dankner WM - Abdominal tuberculosis in children: review of 26 cases. J. Pediatr. Surg. 1996;31:170-6. 